\title{
SIGNIFICANCIA PRÁCTICA DE RESULTADOS NO PARAMÉTRICOS: APORTES A "SATISFACCIÓN E ÍNDICE DE MASA CORPORAL Y SU INFLUENCIA EN EL AUTOCONCEPTO FÍSICO”
}

César Merino-Soto (1) y Marisol Angulo-Ramos (2)

(1) Universidad de San Martín de Porres. Lima. Perú.

(2) Universidad Los Angeles de Chimbote. Chimbote. Perú.

Los autores declaran que no existe ningún conflicto de interés.

\section{Señora Directora:}

En un reciente estudio sobre el autoconcepto físico ${ }^{(1)}$, se reportaron resultados sobre su variabilidad respecto a la satisfacción y el índice de masa corporal. Uno de los aciertos metodológicos del estudio fue la inclusión en la tabla 2 de la fuerza de la relación entre las variables analizadas, expresada mediante el coeficiente V de Cramer. Pero esta referencia fue inconsistente y limita la información que se puede extraer del estudio. Específicamente, los resultados de la hipótesis nula en las tablas 3 y 4 ganarían más contenido interpretativo si se incluyera una estimación de la magnitud o tamaño de las diferencias, algo habitualmente conocido como "significancia práctica". La significancia práctica puede estimarse para el tamaño o magnitud de las diferencias entre los grupos o de las relaciones entre variables estu$\operatorname{diadas}^{(2,3,4,5,6)}$. Dado que en áreas de la ciencia tan heterogéneas como la antropología física $^{(2)}$, la cirugía ósea ${ }^{(3)}$, la epidemiología ${ }^{(4)}$, la pediatría localizada ${ }^{(5)}$ o los problemas de aprendizaje $^{(6)}$ aún está vigente la insistencia en moderar el uso de las pruebas de significancia estadística como el elemento sustancial para interpretar la importancia de los resultados $^{(4,5)}$, la significancia práctica en los resultados de Fernandez ${ }^{(1)}$ deben ser fortale- cidos. Por este motivo, la presente carta complementa los resultados de las tablas 3 y 4 de dicho artículo mediante dos estimadores aplicados a los resultados de la prueba de Kruskal-Wallis: eta ${ }^{2(7)}$ y epsilón ${ }^{2(8)}$. Ambos se interpretan como coeficientes correlacionales, específicamente como varianza compartida entre las dos variables relacionadas ${ }^{(7,8,9)}$. Las estimaciones de magnitud del efecto aplicables para las pruebas no paramétricas generalmente toman la forma de varianza explicada o correlaciones elevadas al cuadrado.

Para facilitar su interpretación, eta y épsilon fueron transformados en coeficientes de correlación, mediante la raíz cuadrada de sus valores. Los resultados de estos reanálisis se presentan en la tabla 1 . Se observa que los resultados estadísticamente significativos obtenidos en el estudio de Fernández (por ejemplo, él rechazó la hipótesis nula) mostraron lo que pueden considerarse pequeños coeficientes. Estas diferencias difícilmente pueden denominarse como realmente significativas o de importancia práctica, y los autores deben contextualizar esta interpretación de acuerdo a las implicaciones clínicas o científicas de su estudio. Esta concluye que los resultados de la significancia estadística deben acompañarse siempre de estimaciones de la magnitud o tamaño del efecto. 


\begin{tabular}{|c|c|c|c|c|}
\hline \multicolumn{5}{|c|}{$\begin{array}{c}\text { Tabla 1 } \\
\text { Estimaciones de magnitud de la relación } \\
\text { "Autoconcepto físico" con "obesidad" } \\
\text { e "insatisfacción laboral". }\end{array}$} \\
\hline \multirow{3}{*}{ Variables } & \multicolumn{4}{|c|}{ Autoconcepto físico } \\
\hline & \multicolumn{2}{|c|}{ Obesidad } & \multicolumn{2}{|c|}{$\begin{array}{l}\text { Insatisfacción } \\
\text { corporal }\end{array}$} \\
\hline & $\begin{array}{l}E t a^{2} \\
(\mathrm{r})\end{array}$ & $\underset{(\mathrm{r})}{\text { Epsilon }^{2}}$ & $\begin{array}{c}E t a^{2} \\
(\mathrm{r})\end{array}$ & $\begin{array}{c}\text { Epsilon }^{2} \\
\text { (r) }\end{array}$ \\
\hline $\begin{array}{l}\text { Habilidad } \\
\text { física }\end{array}$ & $\begin{array}{c}0,001 \\
(0,032)\end{array}$ & $\begin{array}{c}0,005 \\
(0,071)\end{array}$ & $\left(\begin{array}{c}0,0001 \\
(0,001)\end{array}\right.$ & $\begin{array}{c}0,004 \\
(0,063)\end{array}$ \\
\hline $\begin{array}{l}\text { Condición } \\
\text { física }\end{array}$ & $\underline{0,003}$ & $\underline{0,007}$ & $\left(\begin{array}{c}0,002 \\
(0,045)\end{array}\right.$ & $\begin{array}{c}0,005 \\
(0,071)\end{array}$ \\
\hline Fuerza & $\begin{array}{c}0,003 \\
(0,055)\end{array}$ & $\begin{array}{c}0,0001 \\
(0,001)\end{array}$ & $\left(\begin{array}{c}0,002 \\
(0,045)\end{array}\right.$ & $\begin{array}{c}0,001 \\
(0,032)\end{array}$ \\
\hline $\begin{array}{l}\text { Actividad } \\
\text { física } \\
\text { general }\end{array}$ & $\underline{0,014}$ & $\underline{\underline{0,018}} \underline{(0,134)}$ & $\underline{\underline{0,004}} \underline{\underline{(0,063)}}$ & $\underline{\underline{0,008}} \underline{\underline{(0,089)}}$ \\
\hline $\begin{array}{l}\text { Actividad } \\
\text { general }\end{array}$ & $\frac{0,016}{(0,126)}$ & $\frac{0,019}{(0,138)}$ & $\underline{0,007}$ & $\underline{0,011}$ \\
\hline $\begin{array}{l}\text { Atractivo } \\
\text { físico }\end{array}$ & $\frac{0,007}{(0,084)}$ & $\underline{0,011}$ & $\begin{array}{c}0,003 \\
(0,055)\end{array}$ & $\begin{array}{c}0,001 \\
(0,032)\end{array}$ \\
\hline \multicolumn{5}{|c|}{$\begin{array}{l}\text { Valores subrayados: resultados H Kruskall- } \\
\text { Wallis estadísticamente significativos; valores } \\
\text { entre paréntesis: raíz cuadrada de los valores } \\
\text { Eta }^{2} \text { y Epsilon }\end{array}$} \\
\hline
\end{tabular}

\section{BIBLIOGRAFÍA}

1. Fernández Guerrero M, Feu Molina S, Suárez Ramírez M, Suárez Muñoz A. Satisfacción e índice de masa corporal y su influencia en el autoconcepto físico. Rev Esp Salud Pública. 2019;93: 14 de agosto e201908058.

2. Smith RJ. The continuing misuse of null hypothesis significance testing in biological anthropology. Am. J. Phys. Anthropol. 2018; 166(1): 236-245. https://doi.org/10.1002/ ajpa.23399.

3. Gagnier J, Morgenstern H. Misconceptions, misuses, and misinterpretations of $\mathrm{p}$ values and significance testing. $\mathrm{J}$ Bone Joint Surg Am. 2017; 99(18):1598-1603. https://doi. org/10.2106/JBJS.16.01314.

4. Stang A, Poole C, Kuss O. The ongoing tyranny of statistical significance testing in biomedical research. Eur J Epidemiol. 2010; 25(4): 225-230. https://doi.org/10.1007/ s10654-010-9440-x.

5. Pineda S, Sirota M. Determining significance in the new era for $\mathrm{p}$ values. J Pediatr Gastroenterol Nutr. 2018; 67(5): 547-548. doi: https://doi.org/10.1097/ MPG.0000000000002120.

6. Travers JC, Cook BG, Cook L. Null hypothesis significance testing and $\mathrm{p}$ values. Learning Disabilities Research \& Practice (LDRP). 2017; 32(4); 208-215. https://doi. org/10.1111/ldrp.12147.

7. Tomczak M, Tomczak E. The need to report effect size estimates revisited. An overview of some recommended measures of effect size. 2014; Trends in Sport Sci; 1(21): $19-25$.

8. Kelley TL. An unbiased correlation ratio measure. Proceedings of the National Academy of Sciences of the United States of America. 1935; 21(9): 554-559.

9. Kerby DS. The simple difference formula: an approach to teaching nonparametric correlation. Comprehensive Psychology. 2014; 3(1). https://doi.org/10.2466/11.IT.3.1. 